\title{
Satisfacción e importancia de las competencias adquiridas en la universidad según la perspectiva de graduados y de ocupadores: una primera aproximación*
}

\author{
María Cervini-Plá \\ Jaume García \\ Universitat Pompeu Fabra
}

\section{Resumen}

El desarrollo del Espacio Europeo de Educación Superior ha situado el desarrollo de las competencias como un elemento clave en la orientación formativa de las universidades. En este trabajo se pretende efectuar una primera aproximación, tanto desde la perspectiva de los titulados como de los ocupadores, a los niveles de satisfacción con la formación recibida en las universidades y a la relevancia de las diferentes competencias en el mercado de trabajo. Con datos de las encuestas realizadas por la Agència per a la Qualitat del Sistema Universitari de Catalunya, se ha podido establecer que las diferencias entre los niveles y la utilidad de las competencias adquiridas que perciben los titulados parecen estar relacionadas con características sociodemográficas de los mismos y de los estudios realizados. Por otra parte, las características de las empresas explican una parte no muy importante de la variabilidad de la satisfacción e importancia que los ocupadores otorgan a las competencias de los titulados que contratan. Finalmente, la relevancia para el mercado de trabajo de las distintas competencias no se valora de igual manera, según se analice desde la perspectiva de los titulados o de los ocupadores.

Palabras clave: enseñanza, competencias, Espacio Europeo de Educación Superior, graduados, satisfacción.

Clasificación JEL: I20, I21, J24, J28.

\begin{abstract}
The development of the European Higher Education Area has placed the development of competences as a key element in the formative orientation of universities. This paper try to do a first approximation, both from the graduate and employer perspective, to the levels of satisfaction with the training received in the universities and the relevance of the different competencies in the labour market. With data from the surveys carried out by the Agència per a la Qualitat del Sistema Universitari de Catalunya, it has been possible to establish that the differences between the levels and the usefulness of the competences acquired by the graduates seem to be related to their sociodemographic characteristics and to the type of studies. On the other hand, the

* Los autores desean agradecer los valiosos comentarios y recomendaciones de un evaluador anónimo $\mathrm{y}$, a la Agència per a la Qualitat del Sistema Universitari de Catalunya (AQU), el acceso a las bases de datos utilizadas en este estudio. Asimismo, María Cervini-Plá desea agradecer la financiación de los proyectos ECO2017-82350-R y 2017 SGR 558 (grupo GRILMD). Por su parte, Jaume García también desea agradecer la financiación a través de la beca MINECO-ECO2017-83668-R. Cualquier error es exclusiva responsabilidad de los autores.
\end{abstract}


characteristics of the companies explain just a small part of the variability of the satisfaction and importance that the employers give to the competences of the graduates they hire. Finally, the relevance for the labour market of the different competences is not valued in the same way, analysed from the perspective of the graduates or the employers.

Keywords: education, competences, European Higher Education Area, college graduates, satisfaction.

JEL classification: I20, I21, J24, J28.

\section{Introducción}

En los últimos años, las universidades españolas se han adaptado al Espacio Europeo de Educación Superior ${ }^{1}$. Esto implica un cambio de paradigma en el aprendizaje, donde ahora el sistema de evaluación se basa en las competencias (Sánchez, 2008). Fernández-Salinero (2006) explica que el concepto de competencia cambia la orientación de la universidad, tradicionalmente separada del mundo laboral o en paralelo al mismo, y, actualmente, dirigida a dar respuesta a necesidades presentes y futuras que provienen del entorno de trabajo.

Como plantean Hernández et al. (2009), uno de los retos más importantes con los que se enfrenta la sociedad actual y futura es la construcción del aprendizaje y la formación en competencias de acción ${ }^{2}$. En el nuevo discurso político de la Comisión Europea, la construcción del aprendizaje y el desarrollo de las competencias se perciben como una de las mejores inversiones para el desarrollo del capital humano.

Por competencias, como plantea Perrenoud (2004), se entiende la selección y combinación pertinente de conocimientos, habilidades, destrezas, actitudes, valores y normas, que permiten dar respuesta a una situación compleja en un contexto determinado. Por ello, no es suficiente con tener conocimientos (aunque, sin duda, es imprescindible), sino que se necesita dar un paso más: saber aplicarlos con sentido y reflexionar sobre el proceso que se sigue.

En ese sentido, es interesante tener en cuenta que la palabra competencia tiene un significado muy amplio incluyendo, por una parte, las competencias transversales o genéricas y, por otra, las competencias específicas. Las competencias transversales o genéricas son aquellas que rebasan los límites de una disciplina para desarrollarse potencialmente en todas ellas, es decir, son los atributos que debe tener un graduado universitario con independencia de su titulación. Asimismo, son habilidades necesarias para ejercer eficazmente cualquier profesión. Aunque algunas competencias transversales se trabajan en el marco de ciertas asignaturas, no es frecuente que se consideren de forma explícita en una asignatura determinada, sino que deben adquirirse de forma global a lo largo de un grado (Baños y Pérez, 2005). Por otro lado, las

${ }^{1}$ El Espacio Europeo de Educación Superior ha sido desarrollado a través de varios acuerdos firmados entre los ministros de Educación de los diferentes países de la Unión Europea, entre los que podemos destacar, como el más importante, la Declaración de Bolonia de 1999.

2 ECHEVERRÍA (2002) define las competencias de acción como una mezcla de saber, saber hacer, saber estar y saber ser. Entendida también como la capacidad para movilizar los saberes. 
competencias específicas se definen como los atributos que deben adquirir los futuros graduados durante la estancia en la universidad y deben ser definidas, de acuerdo a las especificidades de la propia titulación, por la experiencia de la propia titulación.

El objetivo de este trabajo es analizar la satisfacción e importancia de las competencias adquiridas por los graduados durante la titulación. Con ese objetivo, se utilizan las encuestas de inserción laboral de los titulados en las universidades catalanas realizadas por la Agència per a la Qualitat del Sistema Universitari de Catalunya (AQU), las cuales incluyen información sobre la percepción de los titulados de las competencias adquiridas y su utilidad en sus trabajos. Asimismo, se utilizará la información sobre el estudio de ocupadores, que también realiza dicha agencia, y que permite disponer de información de la satisfacción y la importancia que las empresas otorgan a las competencias adquiridas por los titulados que contratan. En concreto, se utilizarán la encuesta a titulados (AQU, 2014) y el estudio de ocupadores (AQU, 2015) correspondientes a 2014.

En consecuencia, por una parte, a partir de las encuestas realizadas a los propios graduados, se explora en qué áreas existe una mayor diferencia positiva (superávit) o negativa (déficit) entre su valoración subjetiva del nivel y la utilidad de las diferentes competencias. Asimismo, trataremos de aproximar la relación existente entre este déficit o superávit de competencias y algunas características personales y de la titulación de los individuos.

Por otra parte, a partir del estudio de los ocupadores, se analizará el nivel de satisfacción global con las competencias de los recién titulados contratados y cuáles son las competencias que las empresas valoran como más importantes. Asimismo, se tratará de relacionar, tanto la satisfacción como la importancia de las competencias, con características de las empresas o de los ámbitos de las titulaciones de los graduados que contratan.

Salvo error, este es el primer artículo que intenta profundizar en el análisis de las competencias utilizando estas bases de datos. Sin embargo, la riqueza de las mismas ha permitido realizar otros análisis relacionados. Por ejemplo, en un artículo reciente, Di Paolo (2016) analiza la satisfacción con los trabajos obtenidos entre los graduados catalanes que tienen un Doctorado. En otro artículo, Di Paolo y Mañé (2014) analizan las consecuencias de la sobreeducación entre los graduados con Doctorado. Fachelli y Planas (2011) estudian la movilidad intergeneracional de los titulados universitarios catalanes usando la encuesta para el año 2008. Por su parte, García Montalvo (2009) analiza la sobrecualificación de los graduados utilizando varias bases de datos, entre ellas usa las bases de inserción laboral de la AQU para los años 2000, 2004 y 2008.

La estructura del artículo es la siguiente: en la sección dos se presenta una descripción de los datos utilizados y la selección de la muestra utilizada; en la sección tres se describe la metodología empleada en el análisis empírico; en la cuarta sección se presentan los principales resultados; y por último, en la sección cinco, se plantean unas consideraciones finales. 


\section{Datos y selección de la muestra}

Como ya se ha mencionado en la introducción, en esta investigación se utilizan los datos provenientes de las encuestas sobre inserción laboral y las del estudio de ocupadores, realizadas por la Agència per a la Qualitat del Sistema Universitari de Catalunya $(\mathrm{AQU})^{3}$. Estas encuestas surgen debido al interés de las universidades catalanas, tanto públicas como privadas, por conocer la calidad de la inserción en el mercado de trabajo de los recién titulados. La AQU analiza la inserción laboral de los graduados universitarios desde la perspectiva de los titulados cada tres años desde el año 2002 y desde la perspectiva de los ocupadores en el año 2014 ${ }^{4}$. No se han considerado titulados en máster o doctorado.

Con el objetivo de trabajar simultáneamente con las dos bases de datos, se ha seleccionado el año 2014, ya que es el único año para el que se dispone de esta encuesta para ocupadores. Por lo que hace referencia a la encuesta de titulados ${ }^{5}$, estos obtuvieron su título universitario en el curso 2009-10, mientras que el estudio de ocupadores $^{6}$ hace referencia a titulados universitarios contratados recientemente.

Para los titulados universitarios la encuesta de inserción laboral ofrece información sobre sus estudios, datos sociodemográficos, aspectos relacionados con la inserción laboral y un análisis sobre la valoración subjetiva del nivel y la utilidad de competencias adquiridas durante los estudios de cara al trabajo. La base de graduados contiene 15.974 observaciones. En primer lugar, se seleccionan a los menores de 35 años, a fin de analizar únicamente las personas que han realizado una titulación en edades jóvenes. En segundo lugar, se seleccionan a los graduados que han realizado o realizan trabajos para los cuales era necesario tener una titulación universitaria en general o una titulación universitaria específica. No se consideran los individuos que han trabajado o trabajan en actividades para las que no es necesaria la titulación universitaria ${ }^{7}$. Cuando se utilizan estos criterios de selección, la muestra se reduce a 10.544 individuos.

Como se puede observar en el Cuadro 1, el 59,84 por 100 de los graduados que tenemos en la muestra son mujeres. En relación a la situación socioeconómica de los titulados, el 43,3 por 100 de los mismos tiene uno de los dos o los dos padres con estudios superiores. Mientras que para el resto, sus padres tienen un nivel inferior de educación, repartiéndose casi en partes iguales los que tienen los dos padres con estudios primarios o sin educación y los que tienen uno de los dos o los dos padres con estudios medios.

${ }^{3}$ La Generalitat de Catalunya crea esta agencia en 1996 con el objetivo de promover la calidad y la mejora de las universidades catalanas (www.aqu.cat).

${ }^{4}$ En la actualidad se está implementando una nueva encuesta desde la perspectiva de los ocupadores.

${ }^{5}$ La muestra representa el 55,43 por 100 de la población con un error muestral del 0,51 por 100.

${ }^{6}$ No se trata de una muestra representativa de las empresas catalanas. Tal y como se indica en AQU (2015b), están sobrerrepresentadas las medianas y grandes empresas.

7 Este criterio de selección muestral obedece al hecho de que las competencias enseñadas durante los estudios claramente están enfocadas a las personas que luego desempeñarán trabajos que requieran una titulación general o una específica. 


\section{CUADRO 1}

\section{CARACTERÍSTICAS SOCIODEMOGRÁFICAS DE LOS TITULADOS UNIVERSITARIOS}

\begin{tabular}{|c|c|c|c|}
\hline Característica & $\%$ & Característica & $\%$ \\
\hline Sexo & & Tipo de universidad & \\
\hline Mujeres & 59,84 & Pública & 74,19 \\
\hline Hombres & 40,16 & Privada y no presencial & 25,81 \\
\hline Estudios de los padres & & Situación laboral durante la titulación & \\
\hline Los dos estudios primarios o sin estudios & 28,40 & Estudiante a tiempo completo & 36,20 \\
\hline Uno de los dos o los dos con estudios medios & 28,30 & Trabajando en un trabajo relacionado & 44,43 \\
\hline Uno de los dos o los dos con estudios superiores & 43,30 & Trabajando en un trabajo no relacionado & 19,37 \\
\hline Tipo de titulación & & Situación laboral actual & \\
\hline Grado & 0,15 & Ocupado & 88,67 \\
\hline Diplomaturas & 31,52 & Parado & 8,45 \\
\hline Licenciaturas & 43,81 & Inactivo & 2,88 \\
\hline Ingenierías & 8,61 & & \\
\hline Ingenierías técnicas & 12,54 & Ganancias en el trabajo & \\
\hline Arquitectura & 1,28 & Mileurista & 36,84 \\
\hline Arquitectura técnica & 1,26 & Dosmileurista & 35,64 \\
\hline Títulos propios & 0,83 & Más de dosmileurista & 27,52 \\
\hline \multicolumn{4}{|l|}{ Ámbito de la titulación } \\
\hline Humanidades & 5,75 & & \\
\hline Ciencias Sociales & 44,08 & & \\
\hline Ciencias Experimentales & 7,89 & & \\
\hline Ciencias de la Salud & 17,60 & & \\
\hline Ingenierías y Arquitectura & 24,68 & & \\
\hline
\end{tabular}

FUENTE: Elaboración propia a partir de datos de AQU (2014).

En cuanto al tipo de titulación, se puede observar que la mayoría han realizado hecho una licenciatura, dado que los grados se acaban de implementar en aquella época. En cuanto a los estudios realizados por los titulados, se consideran cinco áreas: Humanidades, Ciencias Sociales y Jurídicas, Ciencias Experimentales, Ciencias de la Salud y, por último, Ingenierías y Arquitectura. El porcentaje mayor de titulados (44,08 por 100) corresponde a Ciencias Sociales y Jurídicas. Asimismo, 
en relación con el tipo de universidad en la que estudiaron, casi el 74 por 100 lo hizo en una universidad pública, mientras el resto lo hizo en una privada o en una no presencial.

Por lo que se refiere a la situación laboral, se puede observar que el 36,2 por 100 de nuestra muestra fueron estudiantes a tiempo completo, un 44,43 por 100 trabajó en un trabajo relacionado con sus estudios y el 19,37 por 100 trabajaba, pero con funciones no relacionadas con sus estudios. En cuanto a su situación laboral actual, el 88,67 por 100 de la muestra trabaja en la actualidad, mientras el 11,33 por 100 no trabaja en estos momentos, pero lo ha hecho en otro momento $(8,45$ por 100 en paro y 2,88 por 100 inactivos). Por último, entre los que trabajan, un 36,84 por 100 son mileuristas, un 35,64 por 100 son individuos que cobraban entre 1.000 y 2.000 euros y el resto tiene un ingreso superior a los 2.000 euros $^{8}$.

En relación a las competencias de los titulados, a los que hayan trabajado o trabajan en la actualidad se les pregunta por la formación recibida en la universidad y, en concreto, por el nivel de las competencias adquiridas durante la titulación y su utilidad para el último trabajo que desempeñan o han desempeñado. En concreto, se utiliza una variable en la cual se valoran las competencias con una escala de 0 a 10 para poder compararla con la valoración de los ocupadores, donde se sigue esa misma escala9 .

Los titulados universitarios valoran 14 competencias. Sin embargo, para poder comparar las competencias que se analizan en la encuesta de graduados y de ocupadores, se han redefinido en diez competencias:

- Formación teórica.

- Formación práctica.

- Habilidades comunicativas.

- Trabajo en equipo.

- Liderazgo.

- Análisis y resolución de problemas.

- Toma de decisiones.

- Creatividad.

- Informática.

- Idiomas.

En el Cuadro 2 se presenta una primera aproximación descriptiva a las diez competencias que se analizan. Se presenta la valoración media (y el ranking) de cada competencia en cuanto al nivel recibido en la universidad y la utilidad para el último trabajo desempeñado.

\footnotetext{
${ }^{8}$ Nótese que la información referente a las competencias solamente existe para las que trabajan o han trabajado, es por eso que hemos eliminado de nuestra base los que nunca han trabajado.

${ }^{9}$ La escala original de la encuesta es de 1 (muy bajo) a 7 (muy bueno), pero AQU la ha redefinido en una escala 0 a 10 para facilitar dicha comparación.
} 


\section{CUADRO 2}

COMPETENCIAS VALORADAS POR LOS GRADUADOS

\begin{tabular}{|l|c|c|c|c|}
\hline & \multicolumn{2}{c|}{ Nivel } & \multicolumn{2}{c|}{ Utilidad } \\
\hline \multicolumn{1}{|c|}{ Nivel/Utilidad } & Media & Ranking & Media & Ranking \\
\hline Trabajo en equipo & 6,94 & 1 & 7,85 & 3 \\
\hline Formación teórica & 6,83 & 2 & 5,77 & 9 \\
\hline Análisis y resolución de problemas & 6,22 & 3 & 8,05 & 1 \\
\hline Habilidades comunicativas & 5,93 & 4 & 7,18 & 4 \\
\hline Toma de decisiones & 5,73 & 5 & 7,87 & 2 \\
\hline Formación práctica & 5,60 & 6 & 5,72 & 10 \\
\hline Informática & 5,23 & 7 & 7,16 & 5 \\
\hline Creatividad & 5,22 & 8 & 6,65 & 6 \\
\hline Liderazgo & 4,75 & 9 & 6,61 & 7 \\
\hline Idiomas & 3,15 & 10 & 6,18 & 8 \\
\hline
\end{tabular}

FUENTE: Elaboración propia a partir de datos de AQU (2014).

NOTA: Los valores de la variable oscilan entre 0 (muy bajo) y 10 (muy bueno).

Como se puede observar en el Cuadro 2, las competencias adquiridas con nivel más alto son el trabajo en equipo, la formación teórica, el análisis y la resolución de problemas y las habilidades comunicativas. Por otra parte, las competencias que los graduados consideran más útiles para el trabajo son el análisis y resolución de problemas, la toma de decisión, el trabajo en equipo y las habilidades comunicativas. El coeficiente de correlación entre las medias es 0,33 y entre los rankings es 0,41, indicativo de cierto desajuste entre ambas variables.

Por lo que se refiere al estudio de ocupadores, en el año 2014 la AQU realizó una encuesta sobre las características de los trabajadores contratados. En particular, se conoce si las empresas han contratado titulados universitarios en los últimos cinco años o no. De las 1.435 empresas que conforman la muestra inicial, el 56,31 por 100 han contratado titulados universitarios en los últimos cinco años (808). Esta será nuestra muestra de empresas ${ }^{10}$.

En el Cuadro 3 se presenta una estadística descriptiva de las principales características de estas empresas y que serán utilizadas en posteriores análisis con la muestra de ocupadores. La evidencia descriptiva pone de manifiesto el carácter no representativo de las empresas de la muestra de ocupadores en relación con el total de empresas catalanas, tal y como se ha destacado anteriormente.

\footnotetext{
${ }^{10}$ Del total de empresas de la muestra el 7,49 por 100 no han respondido a esta pregunta.
} 


\section{CUADRO 3}

\section{CARACTERÍSTICAS DE LAS EMPRESAS DEL ESTUDIO DE OCUPADORES}

\begin{tabular}{|l|r|l|r|}
\hline \multicolumn{2}{|c|}{ Característica } & $\%$ & \multicolumn{2}{c|}{ Característica } & $\%$ \\
\hline Número de trabajadores & & Ámbito titulación & \\
\hline Entre 1 y 9 & 31,78 & Humanidades & 2,80 \\
\hline Entre 10 y 50 & 33,04 & Ciencias Sociales y Jurídicas & 29,43 \\
\hline Entre 51 y 100 & 12,99 & Ciencias Experimentales & 4,97 \\
\hline Entre 101 y 200 & 9,21 & Ciencias de la Salud & 5,10 \\
\hline Más de 200 & 12,99 & Ingenierías y Arquitectura & 57,71 \\
\hline & & & \\
\hline Trabajadores con título universitario & & Tipo de establecimiento & \\
\hline Menos del 20\% & 22,88 & Establecimiento único & 59,46 \\
\hline Entre 20\% y 40\% & 14,38 & Establecimiento no sede & 28,52 \\
\hline Entre 40\% y 60\% & 16,16 & Sede central & 12,02 \\
\hline Entre 60\% y 80\% & 8,36 & & 27,30 \\
\hline Entre 80\% y 100\% & 38,22 & Titularidad de la empresa & \\
\hline & & Privada & 74,97 \\
\hline Rama actividad (AQU) & & Pública & 4,07 \\
\hline Construcción & 3,76 & Otras (semipública) & \\
\hline Industrias de la alimentación & 5,37 & & \\
\hline Industria química y farmacéutica & 6,98 & Formación en la empresa & \\
\hline Resto de la industria & 16,64 & No & \\
\hline Servicios a las empresas & 22,15 & Sí & \\
\hline Medios de comunicación & 6,71 & & $\%$ ventas mercado internacional \\
\hline Servicios al consumidor & 9,96 & & \\
\hline Tecnologías de la comunicación & 7,38 & & \\
\hline Educación, cultura e investigación & 2,55 & & \\
\hline Sanidad y servicios sociales & & & \\
\hline & & & \\
\hline
\end{tabular}

FUENTE: Elaboración propia a partir de datos de AQU (2015).

En el caso de que las empresas hayan contratado recientemente titulados universitarios, se les pregunta ${ }^{11}$ por la importancia de determinadas competencias para el trabajo y el nivel de satisfacción con estas competencias de los contratados. La valoración de las competencias se realiza en una escala de 0 a 10. En el estudio de

\footnotetext{
${ }^{11}$ Los cuestionarios de ocupadores son completados en su mayoría por directores de recursos humanos o directores generales de la empresa. Solamente responden a las preguntas sobre competencias las empresas que hayan contratado graduados en los últimos cinco años.
} 
CUADRO 4

COMPETENCIAS VALORADAS POR LOS OCUPADORES

\begin{tabular}{|l|c|c|c|c|}
\hline \multirow{2}{*}{\multicolumn{1}{|c|}{ Competencias }} & \multicolumn{2}{c|}{ Satisfacción } & \multicolumn{2}{c|}{ Importancia } \\
\cline { 2 - 5 } & Media & Ranking & Media & Ranking \\
\hline Informática & 7,84 & 1 & 8,20 & 2 \\
\hline Trabajo en equipo & 7,47 & 2 & 8,34 & 1 \\
\hline Formación teórica & 7,20 & 3 & 7,19 & 9 \\
\hline Habilidades comunicativas & 6,69 & 4 & 7,91 & 5 \\
\hline Idiomas & 6,65 & 5 & 7,63 & 7 \\
\hline Creatividad & 6,58 & 6 & 8,03 & 4 \\
\hline Análisis y resolución de problemas & 6,52 & 7 & 8,17 & 3 \\
\hline Toma de decisiones & 6,20 & 8 & 7,58 & 8 \\
\hline Formación práctica & 6,16 & 9 & 7,70 & 6 \\
\hline Liderazgo & 5,79 & 10 & 6,58 & 10 \\
\hline
\end{tabular}

FUENTE: Elaboración propia a partir de datos de AQU (2015).

NOTA: Los valores de la variable oscilan entre 0 (muy bajo) y 10 (muy bueno).

ocupadores se analizan 16 competencias. Sin embargo, a fin de poder comparar con las competencias valoradas por los titulados, se utilizan las mismas diez competencias ya analizadas para los graduados. En el Cuadro 4 se presenta la información relativa a la satisfacción y la importancia de las diferentes competencias por parte de los ocupadores.

Por una parte, los ocupadores están más satisfechos con los conocimientos de los graduados en Informática, trabajo en equipo, formación teórica y habilidades comunicativas. Por otra parte, las competencias que consideran más importantes para el trabajo son trabajo en equipo, informática, análisis y resolución de problemas y creatividad. El coeficiente de correlación entre las medias es de 0,58 y el de los rankings es 0,56 . Por tanto, existe más similitud entre las dos valoraciones hechas por los ocupadores que en el caso de las realizadas por los titulados.

\section{Metodología}

En la parte empírica del artículo se realizan tres ejercicios con el objetivo de efectuar una primera aproximación al análisis de las competencias adquiridas en la universidad, tanto desde la perspectiva de los titulados como de los ocupadores, y su relación con características de los propios titulados y de las empresas en las que han sido contratados. 


\section{Superávit o déficit en las competencias de los graduados}

Para realizar el primer ejercicio se considera que los graduados evalúan el «nivel» de la competencia como una proxy de la intensidad con la que les han formado en esas competencias y evalúan la «utilidad» como la importancia de dicha competencia para el último trabajo que han tenido. La diferencia de estas dos variables representaría el superávit (diferencia positiva) o déficit (diferencia negativa) en la competencia correspondiente.

Se realizan estimaciones de modelos que relacionan dicha diferencia con características de los graduados utilizando dos aproximaciones alternativas. En la primera, se utilizan modelos de regresión para cada competencia, donde la variable explicativa es el superávit (diferencia) de una determinada competencia para los diferentes individuos. En concreto, las variables explicativas, que se utilizan, son: sexo, edad, ámbito de la titulación, tipo de titulación, tipo de universidad (púbica o privada), situación laboral, ingresos, nivel de estudios de los padres, si ha trabajado durante la carrera, una variable ficticia que indica si para el trabajo actual o último trabajo era necesario una titulación en general o la titulación específica que habían estudiado y, por último, el nivel de la competencia para ese individuo. De alguna forma, con esta última variable pretendemos captar variables no observables del individuo que hacen que tenga una escala determinada en la valoración y, a su vez, permite atenuar el efecto del sesgo de escala de referencia.

La segunda aproximación está basada en la definición de una variable categórica con tres valores posibles: 1) si la variable superávit es negativa (o sea, si hay déficit), 2) si la variable superávit es igual a 0 (es decir, si el individuo considera que el nivel que tiene en la competencia es exactamente igual que la utilidad que le da a la competencia) y 3) si la variable superávit es positiva (el nivel es superior a la utilidad). Con esta variable categórica se estima un modelo probit ordenado con las mismas variables explicativas que en el caso anterior.

\section{Satisfacción e importancia para los ocupadores de las competencias de los titulados}

Un segundo ejercicio que se realiza, en este caso para los ocupadores, consiste en analizar la relación entre la satisfacción global de los ocupadores con las competencias de los graduados y variables que recogen características de las empresas y del ámbito de las titulaciones en las que mayormente contratan dichas empresas. La satisfacción global se define como la media ponderada de los niveles de satisfacción de las diferentes competencias, en la que la ponderación viene dada por la importancia de cada una de ellas.

Se han utilizado tres ponderaciones. La primera se define a partir de la importancia que cada ocupador otorga a cada una de las diez competencias, calculándose para cada uno de ellos la ponderación, como la proporción que la importancia de una determinada competencia tiene sobre la suma de las valoraciones de la importancia de todas las competencias consideradas. 
También se han utilizado otras dos ponderaciones definidas de la misma manera pero, en lugar de utilizar para cada ocupador la valoración de la importancia dada por él mismo, se han utilizado unas únicas ponderaciones para todos los ocupadores. En un caso están basadas en las medias de las valoraciones de los ocupadores para cada competencia y en el otro caso en las medias de las valoraciones de la utilidad (como proxy de la importancia) facilitadas por los titulados universitarios en la encuesta de inserción laboral.

Con esta variable de satisfacción global se ha estimado un modelo de regresión, donde la variable dependiente es este promedio ponderado de la satisfacción con las competencias y las variables explicativas son: el tamaño de la empresa, el porcentaje de titulados dentro de su plantilla, el ámbito de las titulaciones de los graduados contratados, la rama de actividad de la empresa, el porcentaje que las ventas al extranjero representan sobre el total de ventas y la existencia o no de una política de formación propia en la empresa para los recién graduados.

Comparativa de las valoraciones sobre la importancia de las competencias entre titulados y ocupadores

El objetivo de este último ejercicio es analizar las similitudes en la valoración de las competencias realizada por los graduados y por los ocupadores. En concreto, se compara la utilidad (expresada por los graduados) con la importancia (expresada por los empresarios) bajo el supuesto de que ambas variables representan lo mismo desde diferentes perspectivas. El análisis se desagregará según los ámbitos de conocimiento de las diferentes titulaciones.

\section{Resultados}

En esta sección se pretende analizar con mayor detalle la relación entre el nivel (satisfacción) y la utilidad (importancia) que los graduados (ocupadores) otorgan a las diferentes competencias y algunas características asociadas a los mismos (titulados o empresas), mediante modelos que permitan tener en cuenta las correlaciones entre estas características, pero sin una vocación de identificar efectos causales, sino sencillamente relaciones basadas en la evidencia disponible, es decir, un análisis de correlaciones parciales. Los ejercicios para la muestra de graduados y la de ocupadores se comentan en los dos primeros subapartados de esta sección respectivamente, mientras que en el tercer subapartado se comparan las valoraciones de las competencias en ambas muestras, es decir, desde la perspectiva de los titulados y desde la de los ocupadores. 


\section{GRÁFICO 1}

\section{DÉFICIT EN LAS COMPETENCIAS DE LOS TITULADOS}

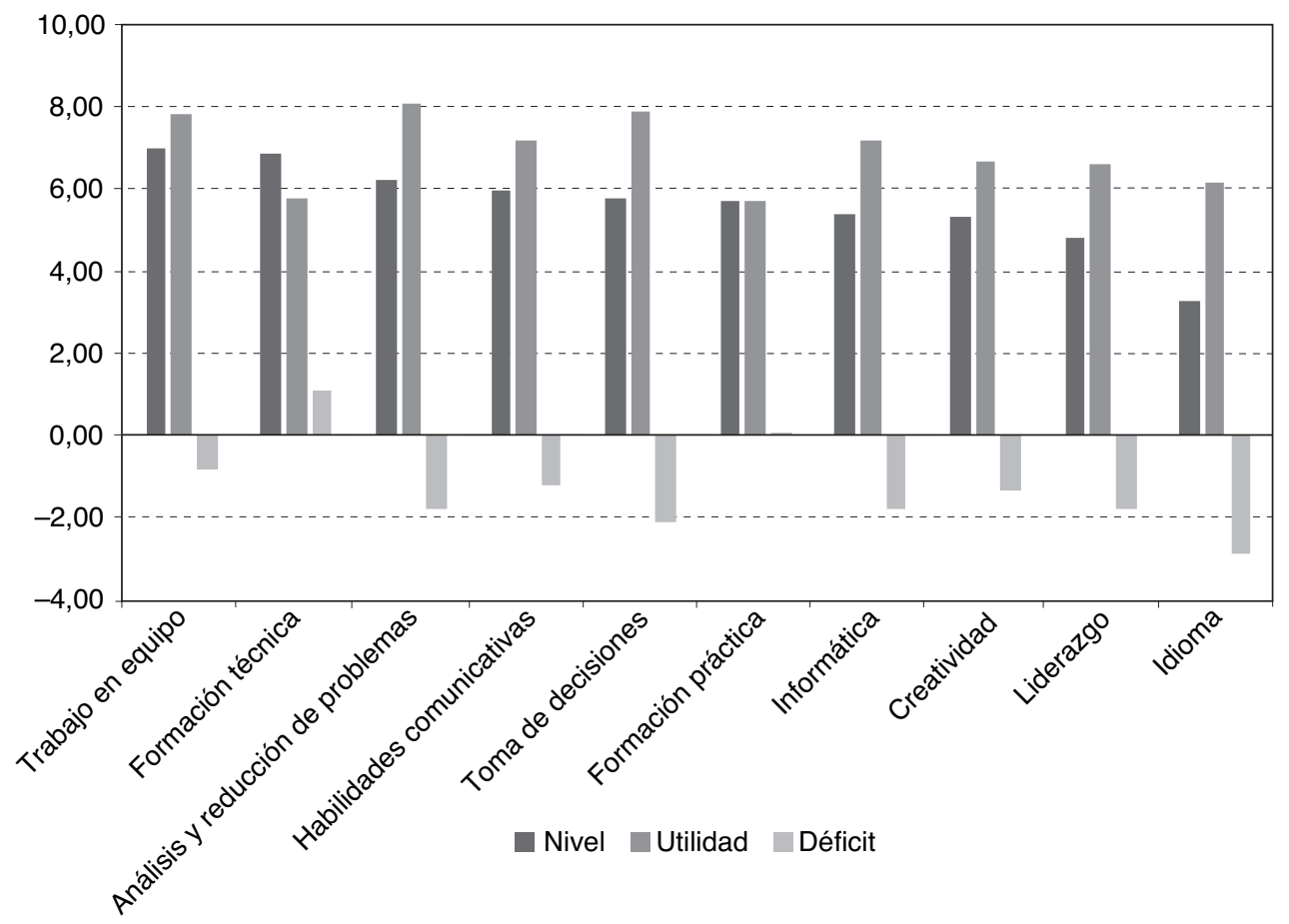

FUENTE: Elaboración propia a partir de datos de AQU (2014).

NOTA: En el eje vertical se reportan, para cada competencia, el valor del promedio del nivel, la utilidad (ambos con valores de 0 a 10) y la diferencia entre el nivel y la utilidad (déficit).

\section{Superávit o déficit en las competencias de los titulados}

En el Cuadro 2 se presentaba la información correspondiente a las medias del nivel y de la utilidad de las competencias según la percepción de los graduados. Haciendo uso de la interpretación dada a dichos conceptos y a la definición de superávit introducida en la sección 3, en el Gráfico 1 se muestran dichos superávits (o déficits) para las diez competencias objeto de análisis, ordenados según el nivel medio de las mismas.

En primer lugar, debe destacarse que todas estas diferencias son significativas al 1 por $100^{12}$, incluso en el caso de la formación básica, competencia para la que la diferencia en valor absoluto es menor. Como puede observarse solo la formación teórica presenta un superávit, mientras que la competencia en idiomas es aquella

12 Tanto en el caso de superávit (diferencia positiva) como de déficit (diferencia negativa), la hipótesis nula es $H_{0}$ : diferencia $=0$, mientras que en el caso de superávit la alternativa es $H_{A}$ : diferencia $>0$, y en el de déficit es $H_{A}$ : diferencia $<0$. 
con un mayor desajuste entre el nivel y la utilidad percibidos por los graduados. Cabe destacar que competencias como la de análisis y resolución de problemas o la formación práctica presentan también déficits importantes, a pesar de que los niveles recibidos están en la parte alta de la distribución.

A fin de analizar la relación entre estas diferencias entre nivel y utilidad de las diferentes competencias, se ha estimado para cada una de las mismas, y con la muestra de graduados, un modelo en el que la variable dependiente es dicha diferencia, medida directamente en términos cuantitativos (modelo de regresión) o mediante una variable cualitativa que toma el valor 1 si hay déficit, el valor 2 si la diferencia es cero y el valor 3 si hay superávit (modelo ordenado ${ }^{13}$ ).

En el Cuadro 5 se presentan los efectos marginales para el grupo de variables con mayor interés desde una perspectiva educativa y sociodemográfica. En el caso de los modelos ordenados, el efecto marginal reportado es la media de los efectos marginales de la correspondiente variable para todos los individuos de la muestra sobre la probabilidad de que se dé un superávit ${ }^{14}$, mientras que en el modelo de regresión el efecto marginal viene dado por el coeficiente de la correspondiente variable. Cabe destacar que los resultados de ambas estimaciones, como era de esperar, apuntan en la misma dirección, aunque utilizan diferente cantidad de información, mayor en el caso de utilizar los valores de las diferencias, aunque a riesgo de cometer algún error de medida, como resultado de que estas variables que miden conceptos como utilidad o satisfacción contienen una componente ordinal, no solo cuantitativa.

De la evidencia presentada en el Cuadro 5 destaca el hecho que, a idénticas características, las mujeres presentan una mayor diferencia entre el nivel y la utilidad percibidos de una competencia (o una mayor probabilidad de percibir un superávit) que los hombres ${ }^{15}$. Ello es así para todas las competencias a excepción de la formación teórica y la formación práctica, para las que la diferencia entre mujeres y hombres no es significativa. Con respecto a la otra variable sociodemográfica, y a pesar de su poca variabilidad, a mayor edad, en general, mayor probabilidad de tener superávit en una determinada competencia o una mayor diferencia entre nivel y utilidad, con una excepción destacada como es la competencia en informática.

Con respecto al tipo de titulación, los licenciados presentan, en general, una mayor diferencia y una mayor probabilidad de superávit en relación a diplomados e ingenieros, con algunas excepciones destacables. Esta diferencia y/o probabilidad es mayor para los ingenieros en el caso de la competencia referida al liderazgo, mien-

${ }^{13}$ Se ha utilizado el modelo Probit ordenado, aunque como es habitual los resultados apenas difieren si se utiliza la versión Logit del mismo.

${ }^{14}$ A fin de simplificar la, ya de por sí, densa estructura del Cuadro 5, se ha optado por no reportar los efectos marginales sobre las probabilidades de los otros dos atributos (déficit y saldo cero), dado que las relaciones entre las diferentes variables y que se dé un tipo de saldo u otro quedan suficientemente recogidas con los efectos sobre la probabilidad de superávit, a pesar de que, si bien el efecto sobre el déficit debe tener signo contrario (sea o no significativo), el efecto sobre saldo cero puede tener uno u otro signo dependiendo del individuo.

${ }^{15}$ Una mayor diferencia significa que, en el caso de superávit, este aumentaría, mientras que, en el caso de una diferencia negativa (déficit), esta disminuiría en valor absoluto. 


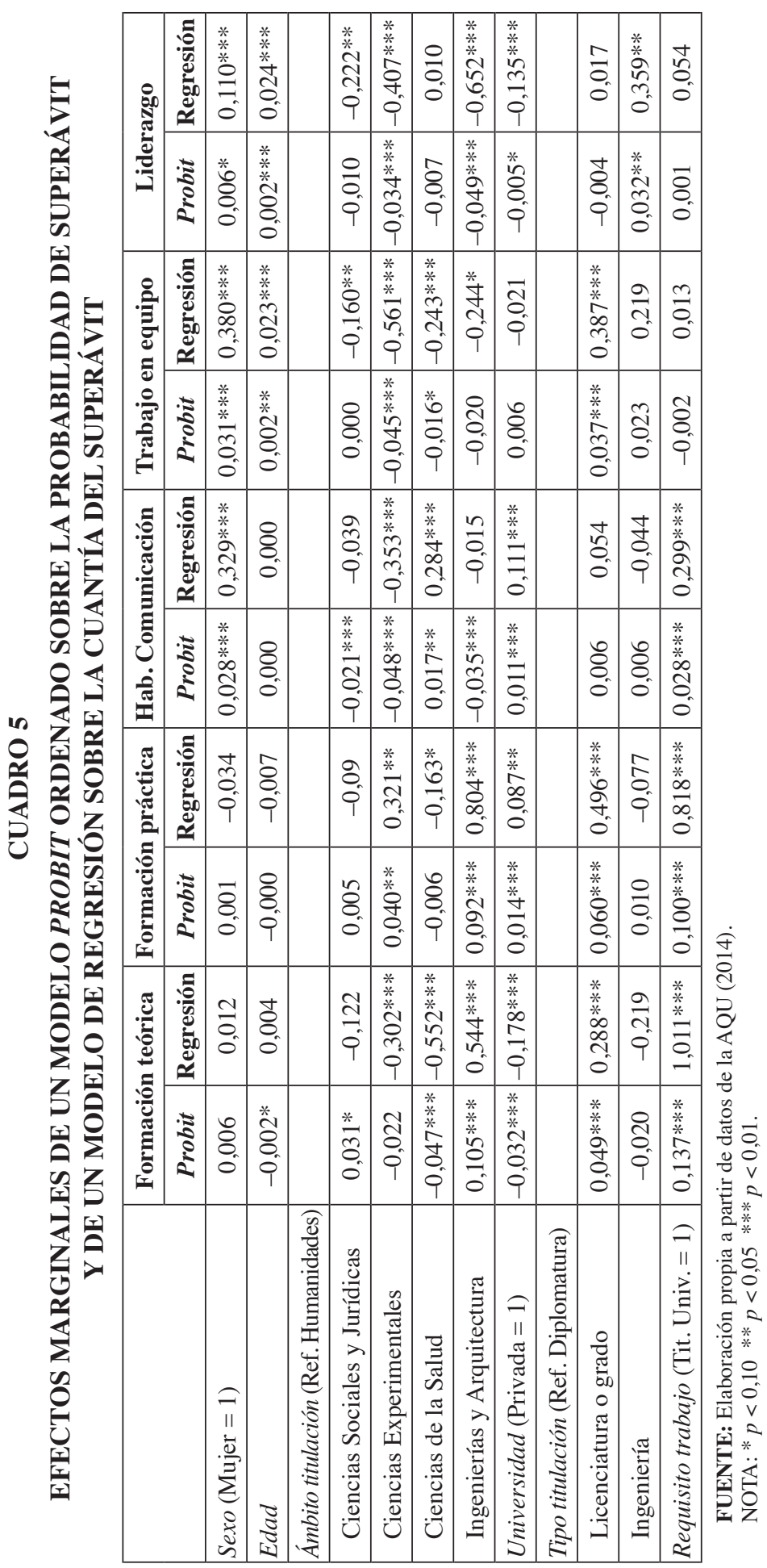




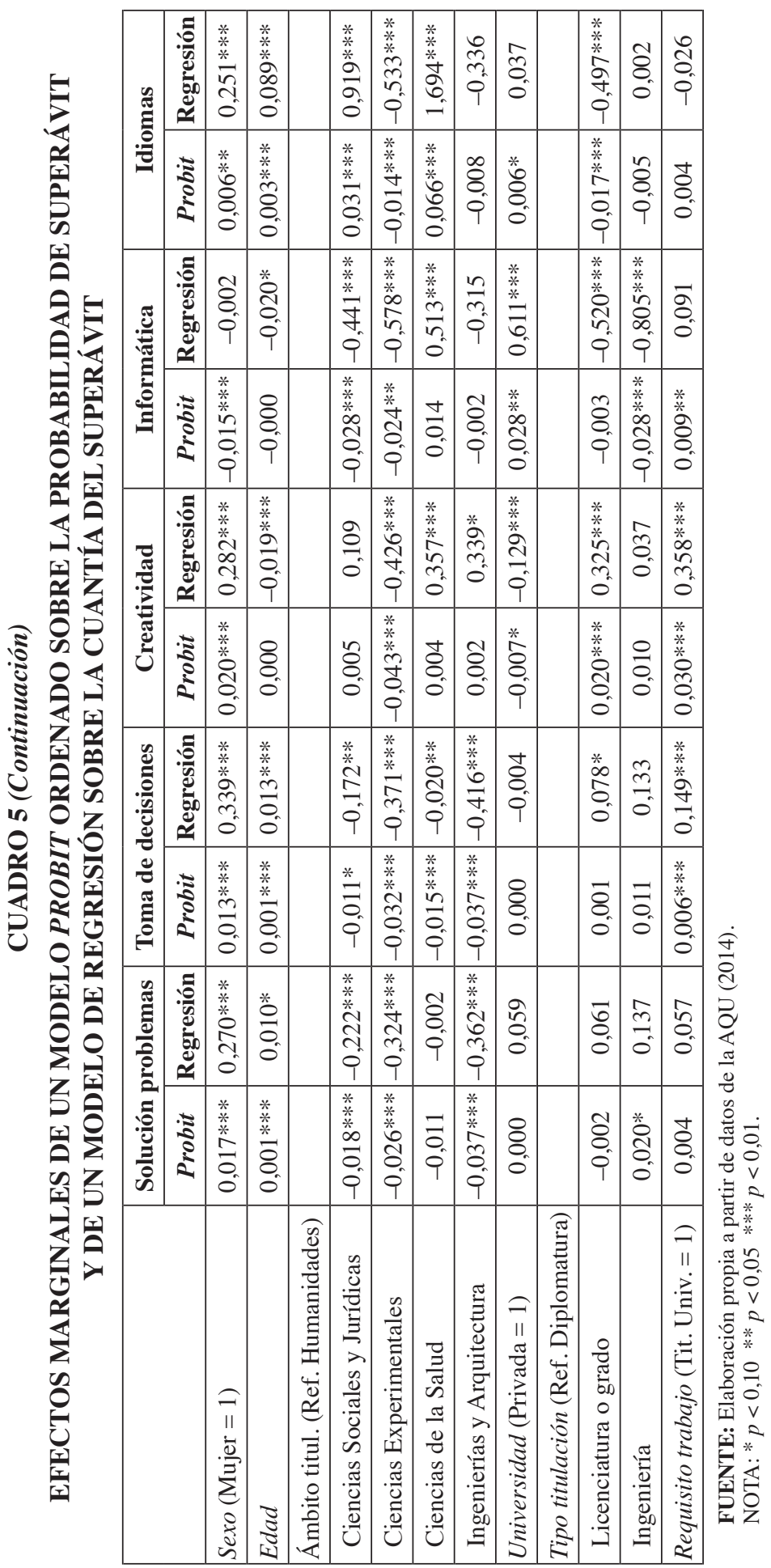


tras que también es más alta para diplomados e ingenieros en caso de los idiomas. En cuanto al ámbito de la titulación la evidencia es heterogénea. Así, en promedio y a idénticas características, las probabilidades de superávit y las diferencias más elevadas se dan en Ciencias de la Salud en cinco de las diez competencias consideradas, en tres en Humanidades y en dos en Ingeniería y Arquitectura. Por el contrario, los casos en que ambas variables toman los valores más bajos se dan en Ciencias Experimentales (5), Ingenierías y Arquitectura (3) y Ciencias de la Salud (2).

Por lo que se refiere a la comparativa entre universidades públicas y privadas, la percepción de los graduados en cuanto a la diferencia entre nivel y utilidad es heterogénea. Para tres competencias (formación práctica, habilidades de comunicación e informática), en promedio y a idénticas características, las universidades privadas presentan probabilidades de superávit y diferencias entre nivel y utilidad más altas. Para otras tres competencias (formación teórica, liderazgo y creatividad) ocurre lo contrario, siendo esos valores más altos en las universidades públicas, no apreciándose diferencias significativas entre universidades públicas y privadas para las otras cuatro competencias.

Finalmente, si el puesto de trabajo que ocupa el graduado requiere formación universitaria, pero no específica de la titulación cursada, esto se traduce en una mayor probabilidad de superávit y/o una mayor diferencia entre nivel y utilidad para la mayoría de competencias, con la excepción de las de trabajo en equipo, liderazgo, análisis y solución de problemas e idiomas, para las que el hecho de que haya o no un requisito de titulación específica para el puesto de trabajo no tiene efecto sobre las variables mencionadas.

Satisfacción e importancia para los ocupadores de las competencias de los titulados

En el caso de los ocupadores, se ha procedido a modelizar separadamente, por un lado, la satisfacción global con las competencias adquiridas en la universidad por los titulados que tienen contratados, y por otro lado, la importancia que otorgan a cada una de las competencias. Todo ello en función de un conjunto de variables que recogen características de la empresa y, en menor medida, de los titulados que tienen contratados.

Dado que la importancia otorgada a cada competencia puede ser sustancialmente distinta según la empresa o según el sector de actividad económica, se ha procedido a realizar una estimación de la satisfacción global del ocupador como media de los niveles de satisfacción, ponderados con el peso de la importancia de la misma, según se ha indicado en la sección anterior.

En el Cuadro 6 se presentan los resultados de la estimación de un modelo de regresión en el que la variable dependiente es la satisfacción global del ocupador con las competencias de los titulados que tiene contratados. La primera cuestión a destacar es la relativamente baja capacidad explicativa del modelo $\left(R^{2}\right.$ ajustado del 


\section{CUADRO 6}

\section{ESTIMACIÓN DE UN MODELO EXPLICATIVO DE LA SATISFACCIÓN GLOBAL DE LOS OCUPADORES}

\begin{tabular}{|c|c|c|c|}
\hline Variable & Coeficiente & Variable & Coeficiente \\
\hline $\begin{array}{l}\text { Número de trabajadores } \\
\text { (Ref. Entre } 51 \text { y 100) }\end{array}$ & & $\begin{array}{l}\text { Ámbito titulación } \\
\text { (Ref. Humanidades) }\end{array}$ & \\
\hline Entre 1 y 9 & $-0,521 * *$ & Cienc. Sociales y Jurídicas & $-0,115$ \\
\hline Entre 10 y 50 & $-0,371 * *$ & Ciencias Experimentales & $-0,022$ \\
\hline Entre 101 y 200 & $-0,372$ & Ciencias de la Salud & $-0,340$ \\
\hline Más de 200 & $-0,535^{* *}$ & Ingenierías y Arquitectura & $-0,170$ \\
\hline \% ventas mercado intnal. & 0,001 & Formación empresa $(=1)$ & $-0,216$ \\
\hline $\begin{array}{l}\text { Trabajadores con título univ. (\%) } \\
\text { (Ref: menos del 20\%) }\end{array}$ & & $\begin{array}{l}\text { Tipo de establecimiento } \\
\text { (Ref: establecimiento único) }\end{array}$ & \\
\hline Entre $20 \%$ y $40 \%$ & 0,000 & Establecimiento no sede & $-0,314 * *$ \\
\hline Entre $40 \%$ y $60 \%$ & 0,073 & Sede central & $-0,331 *$ \\
\hline Entre $60 \%$ y $80 \%$ & $-0,205$ & & \\
\hline Entre $80 \%$ y $100 \%$ & 0,061 & & \\
\hline $\begin{array}{l}\text { Rama actividad (AQU) } \\
\text { (Ref: Sanidad y serv. soc.) }\end{array}$ & & $\begin{array}{l}\text { Titularidad de la empresa } \\
\text { (Ref: privada) }\end{array}$ & \\
\hline Construcción & $1,217 * *$ & Pública & 0,360 \\
\hline Industrias alimentación & $1,039 * *$ & Otras (semipública) & $1,913 * * *$ \\
\hline Ind. química y farmac. & 0,680 & & \\
\hline Resto de la industria & $0,824 *$ & Constante & $6,830 * * *$ \\
\hline Servicios a las empresas & 0,455 & & \\
\hline Medios de comunicación & $0,851 *$ & $\mathbf{R}^{2}$ ajustado & 0,082 \\
\hline Servicios al consumidor & $0,920 * *$ & & \\
\hline Tecnologías comunicación & $0,832 *$ & & \\
\hline Educación, cultura e inv. & $0,969 * *$ & & \\
\hline
\end{tabular}

FUENTE: Elaboración propia a partir de datos de AQU (2015).

NOTA: $* p<0,10 * * p<0,05 * * * p<0,01$.

8,2 por 100), que se encuentra algunas veces al trabajar con microdatos habitual cuando se utilizan microdatos, $y$, en particular, en este caso por el hecho de que la heterogeneidad no observada es muy grande, en la medida en que el nivel de satisfacción depende específicamente de los titulados contratados por cada empresa y las características de estos no están disponibles en la encuesta. Por otra parte, los resultados obtenidos apenas difieren si, en lugar de utilizar las ponderaciones provenientes 
de las respuestas de cada ocupador, se utilizan las medias de la importancia de las competencias en la muestra de ocupadores o las medias de la utilidad que los titulados asignan a cada competencia en la encuesta de graduados.

$\mathrm{Si}$ bien es cierto que algunas de las variables características de la empresa no parecen tener efecto significativo sobre el nivel de satisfacción global (presencia internacional, presencia de titulados universitarios o desarrollo de actividades de formación), sí que se estiman algunas pautas de relación significativas para otros elementos característicos de la empresa. Así, el tamaño de la empresa parece tener un efecto en forma de U invertida sobre el nivel de satisfacción global. A idénticas características, las empresas que tienen entre 51 y 100 trabajadores presentan niveles de satisfacción más elevados, que decrecen a medida que el tamaño de la empresa aumenta o disminuye. Asimismo, el tipo de establecimiento tiene también un efecto significativo sobre el nivel satisfacción. Este es más alto a idénticas características en empresas con una única sede, indicativo de otro aspecto de cómo el tamaño de la empresa está relacionado con la satisfacción global ${ }^{16}$.

Como era de esperar, la heterogeneidad entre empresas, parcialmente captada por la rama de actividad de la misma, tiene una cierta relación con los niveles de satisfacción global de los ocupadores. Así, los niveles más elevados, a idénticas características, se dan en el sector de la Construcción, la Industria de la alimentación, el sector de Educación, cultura e investigación, y en el de los Servicios al consumidor. Por el contrario, la rama de Sanidad y servicios sociales presenta los niveles más bajos a idénticas características. Es probable que esta variable esté muy relacionada con los ámbitos de titulación en los que habitualmente contrata la empresa a sus titulados, y ello explique la no significación de esta última variable a la hora de explicar la satisfacción global de los ocupadores.

En relación con la importancia de las diferentes competencias para los ocupadores, se han estimado modelos de regresión explicativos del nivel de importancia de cada una de las diez competencias consideradas. Los resultados de estas estimaciones se recogen en el Cuadro 7. Al igual que en el modelo anterior, los ajustes de todas las ecuaciones son particularmente bajos como consecuencia del peso que la heterogeneidad no observada tiene en este tipo de modelos. Asimismo, debe mencionarse que, a fin de destacar la significación de las variables cualitativas en cada modelo, se ha variado la categoría de referencia para poder captar las diferencias más significativas entre los atributos de cada variable.

A idénticas características, el tamaño de la empresa parece incidir en la importancia de algunas competencias. En concreto, parece tener un efecto en forma de U invertida sobre la importancia de la formación teórica, más importante en las empresas entre 51 y 100 trabajadores, en las que también se valoran más los idiomas. En cambio, las empresas pequeñas de menos de diez trabajadores parecen valorar más

\footnotetext{
${ }^{16}$ El efecto significativo del tipo de titularidad de la empresa, recogido fundamentalmente a través de la categoría de empresas de titularidad semipública, según la definición en AQU (2015), puede deberse a la escasa proporción de empresas en esta categoría.
} 


\section{CUADRO 7}

ESTIMACIONES DE MODELOS EXPLICATIVOS DE LA IMPORTANCIA DE LAS COMPETENCIAS PARA LOS OCUPADORES

\begin{tabular}{|c|c|c|c|c|c|}
\hline & Teóricas & Prácticas & Comunic. & Trab. Eq. & Liderazgo \\
\hline \multicolumn{6}{|l|}{ Número de trabajadores } \\
\hline Entre 1 y 9 & $-0,326$ & $-0,504$ & 0,154 & $-0,188$ & $-0,418$ \\
\hline Entre 10 y 50 & $-0,397 *$ & $-0,386$ & 0,204 & $-0,087$ & $-0,293$ \\
\hline Entre 51 y 100 & - & $-0,722 * *$ & 0,174 & $-0,098$ & $-0,308$ \\
\hline Entre 101 y 200 & $-0,411 *$ & - & - & - & $-0,167$ \\
\hline Más de 200 & $-0,516^{*}$ & $-0,166$ & 0,062 & $-0,160$ & - \\
\hline$\%$ ventas merc. intnal. & $-0,002$ & 0,000 & 0,000 & 0,001 & 0,004 \\
\hline \multicolumn{6}{|l|}{ Trabaj. con título univ. (\%) } \\
\hline Menos del $20 \%$ & - & - & $-0,116$ & 0,069 & 0,373 \\
\hline Entre $20 \%$ y $40 \%$ & $-0,258$ & $-0,184$ & $-0,160$ & 0,277 & 0,407 \\
\hline Entre $40 \%$ y $60 \%$ & $-0,128$ & $-0,014$ & - & 0,013 & 0,327 \\
\hline Entre $60 \%$ y $80 \%$ & $-0,337$ & $-0,045$ & $-0,484$ & - & 0,099 \\
\hline Entre $80 \%$ y $100 \%$ & $-0,199$ & $-0,021$ & $-0,315$ & $-0,058$ & - \\
\hline \multicolumn{6}{|l|}{ Rama actividad (AQU) } \\
\hline Construcción & 0,597 & 0,364 & 0,146 & - & - \\
\hline Industrias alimentación & - & 0,371 & 0,219 & $0,810 * *$ & $-0,486$ \\
\hline Ind. química y farmac. & 0,134 & 0,035 & 0,101 & 0,192 & $-1,006^{*}$ \\
\hline Resto de la industria & $0,812 * *$ & 0,471 & 0,301 & 0,215 & $-0,570$ \\
\hline Instituciones financ. inmob. & $1,021 * *$ & 0,033 & 0,172 & $0,832 * *$ & $-0,542$ \\
\hline Servicios a las empresas & $0,808 *$ & $0,593 *$ & 0,153 & 0,378 & $-0,664$ \\
\hline Medios de comunicación & 0,753 & $1,382 * * *$ & $0,832 * *$ & $0,744 * *$ & $-0,603$ \\
\hline Servicios al consumidor & $0,962 * *$ & 0,516 & $0,557 *$ & 0,422 & $-0,738 *$ \\
\hline Tecnologías comunicación & 0,538 & $1,172 * * *$ & 0,070 & 0,416 & $-0,703$ \\
\hline Educación, cultura e invest. & 0,710 & - & - & 0,354 & $-0,879 *$ \\
\hline Sanidad y servicios sociales & $1,150 * *$ & $1,122 * *$ & 0,264 & 0,668 & $-0,252$ \\
\hline \multicolumn{6}{|l|}{ Ámbito titulación } \\
\hline Humanidades & - & - & 0,224 & 0,139 & $-0,718$ \\
\hline Cienc. Sociales y Jurídicas & 0,158 & 0,418 & $0,566 * * *$ & 0,015 & $-0,203$ \\
\hline Ciencias Experimentales & 0,800 & 0,571 & 0,279 & - & - \\
\hline Ciencias de la Salud & 0,685 & 0,703 & 0,224 & 0,061 & $-0,512$ \\
\hline Ingenierías y Arquitectura & 0,257 & 0,357 & - & 0,049 & $-0,361$ \\
\hline Formación empresa $(=1)$ & 0,127 & $-0,037$ & 0,200 & $0,277 * *$ & 0,084 \\
\hline
\end{tabular}

FUENTE: Elaboración propia a partir de datos de AQU (2015).

NOTA: $* p<0,10$. ** $p<0,05$. *** $p<0,01$. 
CUADRO 7 (Continuación)

ESTIMACIONES DE MODELOS EXPLICATIVOS DE LA IMPORTANCIA DE LAS COMPETENCIAS PARA LOS OCUPADORES

\begin{tabular}{|l|c|c|c|c|c|}
\hline & Teóricas & Prácticas & Comunic. & Trab. Eq. & Liderazgo \\
\hline Tipo de establecimiento & & & & & \\
\hline Establecimiento único & - & - & - & - & - \\
\hline Establecimiento no sede & $-0,272$ & $-0,137$ & $-0,076$ & $-0,240^{*}$ & $-0,280$ \\
\hline Sede central & 1,156 & $-0,405$ & $-0,061$ & $-0,295$ & $-0,477^{*}$ \\
\hline Titularidad de la empresa & & & & & \\
\hline Pública & 0,272 & - & 0,469 & 0,152 & - \\
\hline Privada & - & 0,010 & - & - & 0,364 \\
\hline Otras (semipública) & 1,16 & $1,230^{* *}$ & 0,745 & $0,899 *$ & 0,482 \\
\hline Constante & $6,859^{* * *}$ & $7,241^{* * *}$ & $7,373^{* * *}$ & $7,807 * * *$ & $7,172 * * *$ \\
\hline \multicolumn{7}{|l|}{} \\
\hline$R^{2}$ ajustado & 0,048 & 0,050 & 0,072 & 0,042 & 0,037 \\
\hline
\end{tabular}

FUENTE: Elaboración propia a partir de datos de AQU (2015).

NOTA: $* p<0,10 * * p<0,05 * * * p<0,01$.

\section{CUADRO 7 (Continuación)}

\section{ESTIMACIONES DE MODELOS EXPLICATIVOS DE LA IMPORTANCIA DE LAS COMPETENCIAS PARA LOS OCUPADORES}

\begin{tabular}{|l|c|c|c|c|c|}
\hline & Sol. prob. & Toma dec. & Creatividad & Inform. & Idiomas \\
\hline Número de trabajadores & & & & & \\
\hline Entre 1 y 9 & $0,386^{*}$ & $0,574^{* *}$ & 0,331 & $-0,538^{* *}$ & - \\
\hline Entre 10 y 50 & 0,237 & 0,295 & 0,203 & $-0,382^{*}$ & 0,301 \\
\hline Entre 51 y 100 & - & 0,149 & 0,001 & $-0,178$ & $0,716^{* *}$ \\
\hline Entre 101 y 200 & 0,263 & 0,332 & $0,616^{* *}$ & - & 0,478 \\
\hline Más de 200 & 0,009 & - & - & $-0,324$ & 0,223 \\
\hline \% ventas mercado internacional & 0,001 & 0,001 & $0,005^{*}$ & $-0,000$ & $0,019^{* * *}$ \\
\hline Trabaj.con título universitario $\%$ ) & & & & & \\
\hline Menos del 20\% & 0,262 & 0,378 & $0,604 *$ & $0,780^{* * *}$ & $-0,243$ \\
\hline Entre 20\% y 40\% & 0,227 & $0,501 * *$ & 0,374 & $0,623 * * *$ & $-0,680 *$ \\
\hline Entre 40\% y 60\% & - & $0,465^{* *}$ & $0,743 * *$ & 0,344 & $-0,199$ \\
\hline Entre 60\% y 80\% & 0,245 & 0,203 & - & - & - \\
\hline Entre 80\% y 100\% & 0,215 & - & 0,239 & 0,274 & $-0,267$ \\
\hline
\end{tabular}

FUENTE: Elaboración propia a partir de datos de AQU (2015).

NOTA: $* p<0,10 * * p<0,05 * * * p<0,01$. 


\section{CUADRO 7 (Continuación)}

ESTIMACIONES DE MODELOS EXPLICATIVOS DE LA IMPORTANCIA DE LAS COMPETENCIAS PARA LOS OCUPADORES

\begin{tabular}{|c|c|c|c|c|c|}
\hline & Sol. prob. & Toma dec. & Creatividad & Inform. & Idiomas \\
\hline \multicolumn{6}{|l|}{ Rama actividad (AQU) } \\
\hline Construcción & $-0,334$ & - & $0,878 *$ & $0,673^{*}$ & - \\
\hline Industrias alimentación & $-0,856^{* *}$ & $-0,686$ & $0,834 *$ & 0,525 & $1,347 * *$ \\
\hline Industria química y farmacéutica & $-0,887 * * *$ & $-1,002 * *$ & - & - & 0,837 \\
\hline Resto de la industria & $0,838 * * *$ & $-0,915 * * *$ & 0,361 & 0,369 & $0,931 *$ \\
\hline Instituciones financ. inmob. & $-0,543^{*}$ & $-0,655$ & 0,644 & $1,292 * * *$ & $1,337 * *$ \\
\hline Servicios a las empresas & $-0,551 * *$ & $-0,823 *$ & $0,712 *$ & $1,111 * * *$ & 0,910 \\
\hline Medios de comunicación & $-0,644 *$ & $-0,376$ & $1,054 * *$ & $1,534 * * *$ & 0,322 \\
\hline Servicios al consumidor & $-0,378$ & $-0,702 *$ & $0,81 * *$ & $0,714 * *$ & $1,072 *$ \\
\hline Tecnologías de la comunicación & - & $-0,633$ & $1,180 * * *$ & $1,242 * * *$ & $1,243 * *$ \\
\hline Educación, cultura y ocio & $-1,306^{* * *}$ & $-1,179 * * *$ & 0,600 & 0,332 & $1,323 * *$ \\
\hline Sanidad y servicios sociales & $-0,230$ & $-0,938 *$ & $0,971 * *$ & 0,280 & $1,368 *$ \\
\hline \multicolumn{6}{|l|}{ Ámbito titulación } \\
\hline Humanidades & - & - & $-0,479$ & $-0,667$ & - \\
\hline Ciencias Sociales y Jurídicas & 0,055 & $-0,243$ & $-0,607 *$ & $-0,488$ & $1,076^{*}$ \\
\hline Ciencias Experimentales & 0,040 & $-0,446$ & - & - & 0,765 \\
\hline Ciencias de la Salud & 0,328 & $-0,300$ & $-0,567$ & $-0,416$ & 0,572 \\
\hline Ingenierías y Arquitectura & 0,162 & $-0,309$ & $-0,502$ & $-0,281$ & 0,902 \\
\hline Formación empresa $(=1)$ & 0,173 & $-0,165$ & 0,159 & $-0,177$ & 0,194 \\
\hline \multicolumn{6}{|l|}{ Tipo de establecimiento } \\
\hline Establecimiento único & - & $-0,140$ & $-0,013$ & - & - \\
\hline Establecimiento no sede & 0,051 & - & - & $-0,276^{*}$ & $-0,161$ \\
\hline Sede central & 0,077 & $-0,226$ & $-0,067$ & $-0,551 * *$ & $-0,085$ \\
\hline \multicolumn{6}{|l|}{ Titularidad de la empresa } \\
\hline Pública & - & - & - & - & 0,844 \\
\hline Privada & 0,119 & $-0,077$ & $-0,070$ & $-0,608^{*}$ & 0,89 \\
\hline Otras (semipública) & 0,721 & $-0,328$ & $1,291 * *$ & $-1,019$ & - \\
\hline Constante & $7,841 * * *$ & $8,310 * * *$ & $6,821 * * *$ & $8,512 * * *$ & $4,268 * * *$ \\
\hline$R^{2}$ ajustado & 0,042 & 0,045 & 0,051 & 0,083 & 0,147 \\
\hline
\end{tabular}

FUENTE: Elaboración propia a partir de datos de AQU (2015).

NOTA: $* p<0,10 * * p<0,05 * * * p<0,01$. 
que el resto la capacidad de análisis y resolución de problemas y la toma de decisiones, valorando menos que el resto los conocimientos en informática. En cuanto al tipo de establecimiento, destaca el hecho de que, en aquellos que son la sede central de la empresa, algunas competencias se valoran menos que en otro tipo de establecimientos, en particular, por lo que se refiere al liderazgo. A su vez, la titularidad de la empresa apenas tiene relación significativa con la importancia de las competencias, salvo en el caso de la creatividad.

Las dos variables más ligadas a las estrategias empresariales a distintos niveles (presencia internacional y política de formación propia) tienen una relación significativa con la importancia de las competencias para algunos casos muy específicos. Un mayor porcentaje de las ventas en el extranjero sobre las ventas totales está relacionado positivamente con la importancia de los idiomas y, en menor medida, con la creatividad. Por su parte, el hecho de que la empresa realice formación interna para los recién titulados se relaciona positivamente con la importancia del trabajo en equipo.

$\mathrm{Al}$ igual que en el caso de la satisfacción global, la rama de actividad de la empresa capta una parte significativa de la variabilidad de la importancia de cada competencia, aunque con patrones variados según la competencia, difíciles de resumir. En cualquier caso, cabe destacar que las ramas de Tecnologías de la comunicación y Medios de comunicación están entre las que, a idénticas características, valoran más competencias como formación práctica, creatividad e informática. A su vez, en la rama de Sanidad y servicios sociales, la formación teórica y la formación práctica, junto a idiomas y creatividad están entre las competencias con niveles más elevados, a idénticas características. Por último, y a pesar de la posible asociación con la rama de actividad, el ámbito de la titulación en el que la empresa capta mayoritariamente a sus titulados también tiene algunos efectos significativos en su relación con la importancia de las competencias según los ocupadores. Así, el ámbito de Ciencias Sociales y Jurídicas es el que está más relacionado de manera positiva y significativa con la importancia de las habilidades de comunicación y los idiomas y, en cambio, es el menos relacionado con la creatividad.

\section{Comparativa de las valoraciones sobre la importancia de las competencias entre titulados y ocupadores}

A la hora de comparar la perspectiva de los graduados y los ocupadores, y de acuerdo a como están formuladas las correspondientes preguntas en cada una de las dos encuestas, la importancia que le da la empresa a determinada competencia es equiparable a la valoración de la utilidad de la competencia que expresan los titulados a la utilidad de la competencia que responden los titulados.

En el Cuadro 8 se presenta, para cada una de las competencias, la valoración media de la utilidad de las mismas desde la perspectiva de titulados y su importancia según los ocupadores, así como el ranking de las mismas de acuerdo a dicha valoración media. El coeficiente de correlación entre las medias de ambas valoraciones es 0,49 , al igual que el de los rankings. 


\section{CUADRO 8 \\ UTILIDAD (TITULADOS) E IMPORTANCIA (OCUPADORES) DE LAS COMPETENCIAS}

\begin{tabular}{|l|c|c|c|c|}
\hline \multirow{2}{*}{ Competencias } & \multicolumn{2}{|c|}{ Utilidad (titulados) } & \multicolumn{2}{c|}{ Importancia (ocupadores) } \\
\cline { 2 - 5 } & Media & Ranking & Media & Ranking \\
\hline Análisis y resolución de problemas & 8,05 & 1 & 8,17 & 3 \\
\hline Toma de decisiones & 7,87 & 2 & 7,58 & 8 \\
\hline Trabajo en equipo & 7,85 & 3 & 8,34 & 1 \\
\hline Habilidades comunicativas & 7,18 & 4 & 7,91 & 5 \\
\hline Informática & 7,16 & 5 & 8,20 & 2 \\
\hline Creatividad & 6,65 & 6 & 8,03 & 4 \\
\hline Liderazgo & 6,61 & 7 & 6,58 & 10 \\
\hline Idiomas & 6,18 & 8 & 7,63 & 7 \\
\hline Formación teórica & 5,77 & 9 & 7,19 & 9 \\
\hline Formación práctica & 5,72 & 10 & 7,70 & 6 \\
\hline
\end{tabular}

FUENTE: Elaboración propia a partir de datos de AQU $(2014,2015)$..

NOTA: Los valores de la variable oscilan entre 0 (muy bajo) y 10 (muy bueno).

Cabe destacar que la valoración de las competencias por parte de los ocupadores (entre 6,58 y 8,34) es mucho más homogénea que la de los graduados (entre 5,66 y $8,05)$. Asimismo, estas valoraciones de la importancia por parte de los ocupadores también son mucho más altas que las de los graduados, en la mayoría de casos, y no de manera uniforme. Así, hay cuatro competencias con una diferencia a favor de la importancia otorgada por los ocupadores por encima de la unidad (creatividad, idiomas, formación teórica y formación práctica), mientras que hay solo dos con valores medios más altos para los titulados, pero prácticamente despreciables (toma de decisiones y liderazgo). Esta aparente menor valoración por parte de los titulados puede estar reflejando, entre otros aspectos, una distinta manera de entender la escala de valoración por parte de ocupadores y titulados (sesgo debido a la escala de referencia) o que en la valoración de la utilidad por parte de los titulados también se tienen en cuenta, en algunos casos, aspectos relativos al nivel y a la calidad de la formación recibida en la universidad para la correspondiente competencia.

Este patrón de relación entre las valoraciones de titulados y ocupadores también se reproduce cuando se realiza esta comparativa según los ámbitos de la correspondiente titulación, aunque con una cierta heterogeneidad. En el caso de los ocupadores, las medias de las valoraciones de la importancia agregando todas las competencias, oscilan entre 7,67 y 7,78, mientras que en el caso de las valoraciones de los titulados, estas no solo son inferiores en todos los ámbitos, sino que presentan una mayor hete- 
rogeneidad, oscilando entre 6,61 y 7,07. Asimismo, las correlaciones entre ambas valoraciones (ya sea en nivel o por rankings) difieren de manera notoria entre los cinco ámbitos. En Humanidades, Ciencias Sociales y Jurídicas y Ciencias Experimentales las correlaciones entre las medias se sitúan por encima de 0,55 , mientras que en Ciencias de la Salud e Ingenierías y Arquitectura están por debajo de 0,45.

Finalmente, cabe destacar que para los estudiantes, sea del ámbito que sea, su titulación las competencias asociadas al análisis y resolución de problemas y a la toma de decisiones están entre las tres más valoradas, mientras que para los ocupadores la única siempre aparece entre las tres más valoradas, sea cual sea el ámbito, es el trabajo en equipo.

\section{Consideraciones finales}

En el presente artículo, utilizando las bases de datos de la encuesta de inserción laboral de los graduados y la del estudio de ocupadores, realizadas por la AQU, se han realizado tres ejercicios con el objetivo de analizar la satisfacción e importancia de las competencias adquiridas por los graduados, desde el punto de vista tanto de los titulados como de los ocupadores.

Analizando la diferencia entre el «nivel» y la «utilidad» de las competencias para los titulados, se encuentra que para la mayoría de las competencias el nivel es inferior a la utilidad. Sin embargo, se encuentran diferencias según características: los hombres parecen tener más déficit, todo apunta a que las universidades públicas destacan en algunas competencias y las privadas en otras, y también se encuentran diferencias según la titulación.

En cuanto a los ocupadores, se observa que las características de la empresa no explican suficientemente ni la «satisfacción» ni la «importancia» de las competencias. Sin embargo, se encuentra cierta heterogeneidad por rama y por tamaño de empresa. Variables como el porcentaje de ventas en el mercado internacional o la formación en la empresa son relevantes en la importancia de algunas competencias.

Por último, cuando se explora la semejanza entre la «utilidad» expresada por los titulados y la «importancia» asignada por los ocupadores se encuentra una correlación de 0,49 , aunque esta difiere según el ámbito de estudio. Los valores que otorgan los ocupadores a la «importancia» de las competencias son más altos y con un menor rango de variación que en el caso de la «utilidad».

\section{Referencias bibliográficas}

[1] AGÈNCIA PER A LA QUALITAT DEL SISTEMA UNIVERSITARI DE CATALUNYA (2014). Universitat $i$ Treball a Catalunya 2014. Estudi de la inserció laboral de la población titulada de les universitats catalanes. Agència per a la Qualitat del Sistema Universitari de Catalunya. 
[2] AGÈNCIA PER A LA QUALITAT DEL SISTEMA UNIVERSITARI DE CATALUNYA (2015). Ocupabilitat i competències dels graduats recents: L'opinió de les empreses i institucions. Principals resultats de l'estudi d'Ocupadors 2014. Agència per a la Qualitat del Sistema Universitari de Catalunya.

[3] BAÑOS, J. y PÉREZ, J. (2005). «Cómo fomentar las competencias transversales en los estudios de Ciencias de la Salud: una propuesta de actividades». Educación Médica, 8, 216-225.

[4] DI PAOLO,A. (2016). «(Endogenous) occupational choices and job satisfaction among recent Spanish PhD recipients». International Journal of Manpower, 37, 511-535.

[5] DI PAOLO, A. y MAÑÉ, F. (2014). «Are we wasting our talent? Overqulification and overskilling among PhD graduates». Working Paper XREAP2014-06, Xarxa de Referència en Economia Aplicada (XREAP).

[6] ECHEVERRÍA, B. (2002). «Gestión de la competencia en acción profesional». Revista de Investigación Educativa, 20 (1), 7-43.

[7] FACHELLI, S. y PLANAS, J. (2011). «Equidad y movilidad intergeneracional de los titulados universitarios catalanes». Papers, 96/4, 1307-1331.

[8] FERNÁNDEZ-SALINERO, C. (2006). «Las competencias en el marco de la convergencia europea: Un nuevo concepto para el diseño de programas educativos». Encounters on Education, 7, 131-153.

[9] GARCÍA MONTALVO, J. (2009). «La inserción laboral de los universitarios y el fenómeno de la sobrecualificación en España». Papeles de Economía Española, 119, 172187.

[10] HERNÁNDEZ, F.; MARTÍNEZ, P.; MARTÍNEZ, M. y MONROY, F. (2009). «Aprendizaje y competencias. Una nueva mirada». Revista Española de Orientación y Psicopedagogía, 20, 312-319.

[11] PERRENOUD, P. (2004). Diez, nuevas competencias para enseñar. Barcelona, Graó.

[12] SÁNCHEZ,A. (2008). «Elementos clave en el diseño de módulos y titulaciones EEES». Revista de Investigaciones en Educación, 5, 41-48. 